\title{
Mommy — where do tumors come from?
}

\author{
Ervin H. Epstein Jr. \\ Children's Hospital of Oakland Research Institute, Oakland, California, USA.
}

\begin{abstract}
Identification of the cell of origin of cancers is a hot topic, and recently no fewer than five groups have addressed this question for basal cell carcinomas (BCCs) of the skin. In this issue of the JCI, Grachtchouk et al. present evidence from engineered mouse models that at least some BCCs can arise from hair follicle tissue stem cells. Although these cells sustain oncogenic mutations while in the stem cell niche, they may have to leave that stem cell niche to become tumor cells.
\end{abstract}

Basal cell carcinomas (BCCs), the most common of human cancers, are so named because of their histologic resemblance to basal keratinocytes (1), the cells of the interfollicular epidermis, sebaceous glands, and hair follicle that abut the dermis. In addition to their morphologic resemblance to these normal cells, BCCs also express keratins 5 and 14, which are characteristic of the normal basal layer. However, this histologic resemblance does not necessarily indicate that the cell that sustains the oncogenic mutation(s) leading to the development of the BCC (aka the "cell of origin") is a basal cell, nor does it indicate in which basal compartment this cell might reside. In this issue of the JCI, Grachtchouk et al. weigh in on this question (2), extending their previous work (3) and joining a recent flurry of publications from a number of groups - including our own (4) - that address the cell of origin of BCCs using Cre-lox technology to activate hedgehog $(\mathrm{Hh})$ signaling in various skin cell compartments. The studies used somewhat different models and came to somewhat different conclusions, but if we view the group from a distance, we can come to at least some conclusions.

\section{Why does the cell of origin matter?}

One answer (beyond the joy of discovery that sustains us all through progressive lowering of NIH paylines), is that in general it is really, really hard to cure cancers once they become clinically apparent, and it might be easier to treat them at their very

Conflict of interest: Ervin H. Epstein Jr. has been a paid consultant for Genentech, Novartis, and BMS regarding their Hh inhibitor projects and is the principal investigator of an ongoing investigator-initiated trial of the anti-BCC efficacy of the Genentech $\mathrm{Hh}$ inhibitor GDC-0449 in patients with Gorlin syndrome.

Citation for this article: J Clin Invest. doi:10.1172/ JCI57700. earliest stage. Such treatment implies we can identify cancers early, and it should be easier to do so if we know where they are lurking before they blossom into detectable lesions (5).

Beyond the simple fact of their high incidence and their ready accessibility for analysis, much about BCCs makes them attractive for study. Thus, these cancers are driven by enhanced Hh signaling due to mutations in genes encoding the signaling machinery; $80 \%-90 \%$ of cases are caused by inactivation of an inhibitor of the pathway, the PTCH1 gene, and $10 \%-20 \%$ of cases are caused by activating mutations of the $\mathrm{Hh}$ receptor $S M O$ (1). Also, much is known about the stem cells of the skin, and tools enabling genetic alteration of various cell compartments are available. In addition, a number of murine models have been developed to study BCC carcinogenesis, all relying on activation of Hh signaling.

\section{An innovative mouse model}

To control Hh signaling at will, Grachtchouk et al. engineered mice expressing the mutant GLI2 $\Delta \mathrm{N}$ allele, GLI2 being the transcription factor at the "business" end of the Hh signaling pathway. This mutant is a version that is a particularly strong transcriptional activator. The power of the technique used by this group was that it allowed tissue- and temporal-specific expression of GLI $2 \Delta \mathrm{N}$, and by varying the amount of doxycycline given to the mouse, they also could control the degree of GLI2 $\Delta \mathrm{N}$ expression.

The group first focused their attention on the area containing the best-studied keratinocyte skin stem cells - the follicular bulge, which lies below the sebaceous glands. When they activated GLI2 $\Delta \mathrm{N}$ expression in keratin $15^{+}\left(\mathrm{K} 15^{+}\right)$cells of

\section{Why study BCC?}

the bulge, the mice developed microscopic tumors with the histology and protein expression patterns of BCCs. These tumors developed on all body sites biopsied and became grossly evident on the back skin within three weeks. Grachtchouk et al. then focused in on the lowermost part of the bulge - the secondary hair germ - and found the same results when they activated Gli2 expression only in this site using Lgr5-driven Cre. Activating GLI2 $\Delta \mathrm{N}$ expression more broadly in all basal cell compartments produced hyperplasia within a few days in the follicle, the sebaceous glands, and the interfollicular epidermis, indicating that all of these are capable of responding to GLI $2 \Delta \mathrm{N}$-stimulated transcription. Interestingly, the main part of the bulge - above the secondary hair germ - expressed GLI2 $\Delta \mathrm{N}$ but did not undergo hyperplasia, perhaps because of increased apoptosis at this site; and the follicular isthmus, the part just above the bulge, neither upregulated GLI $2 \Delta \mathrm{N}$ expression nor developed hyperplasia. Taken together, these data suggest that the BCCs seen with three weeks of GLI $2 \Delta \mathrm{N}$ expression in the K15-CrePR1 mice arose from the secondary hair germ, and that the main part of the bulge may be protected from $\mathrm{Hh}$-driven hyperplasia at least in part by an apoptotic response. Finally, they assessed the results of administering lower doses of the tetracycline derivative doxycycline to produce lower expression of GLI2 $\Delta \mathrm{N}$ in basal cells and saw only hyperplasia and tumors appearing less malignant than BCCs, similar to what is seen when the apparently weaker Hh activating mutant Smo allele is expressed and consonant with their previous studies indicating that lower-level activation of Hh signaling produces more benign follicular tumors, whereas higherlevel Hh activation produces tumors that are more BCC-like (6).

\section{Putting these findings into context}

Three other groups have published studies (7-9) using mutant alleles to activate Hh signaling in the study of BCC. Hence, in total, investigators have assessed the effects of activating Smo, Gli1, or Gli2 and of deleting the pathway inhibitor Ptch1 


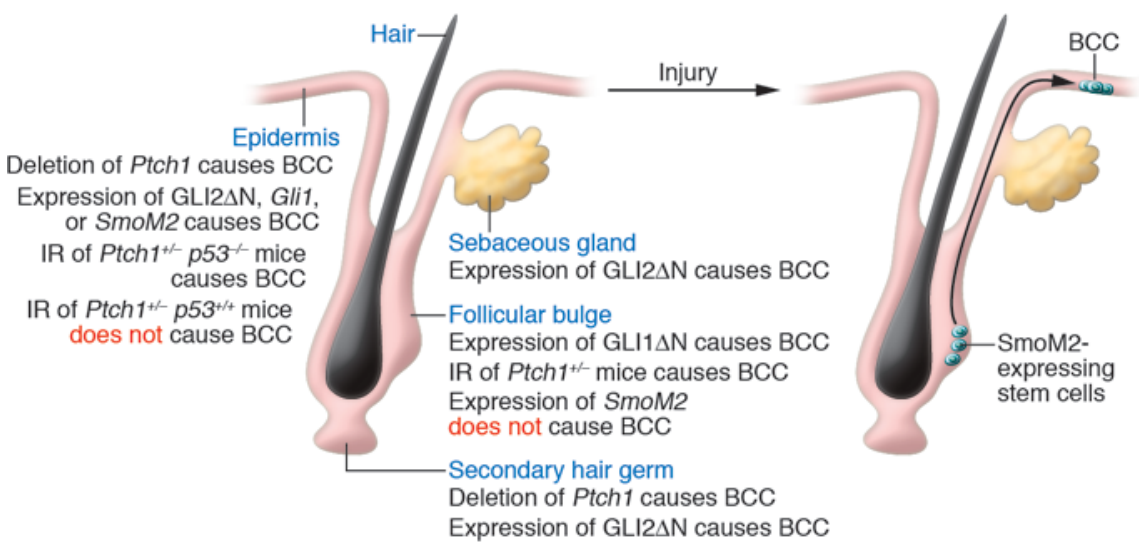

Figure 1

The cell of origin of BCCs differs in mouse models according to the allele producing Hedgehog signaling activation, but at least some tumors arise from the hair follicle bulge stem cells. BCC here refers to an array of tumors of greater or lesser degrees of differentiation and growth potential. IR, ionizing radiation.

(Figure 1). Of interest, two groups $(8,9)$ wounded mouse skin and found more vigorous microscopic tumor development from the newly formed epidermal covering. Various mechanisms can be invoked to explain this stimulation of tumorigenesis by wounding, and the idea of wounding causing the appearance of tumors is well founded in both clinical anecdotes as well as animal models (10). One potential mechanism on which both groups focus is the movement of cells out of the follicle, as occurs normally during skin wound repair. This suggests that the follicle, in particular the bulge, may be relatively resistant to tumorigenesis in these models (perhaps via increased apoptosis) but that once the cell leaves the follicle this inhibition dissipates. Alternatively, wound-associated inflammation could help stimulate tumorigenesis, but at least some other forms of inflammation (e.g., more superficial than full skin thickness wounding, such as hair plucking) appeared not to be stimulatory, suggesting that inflammation per se may not be the mechanism by which wounding stimulates tumorigenesis in their models. Consistent with this idea of movement of cells changing their tumorigenic potential, Grachtchouk et al. found that induction of the hair follicle growth phase (anagen) greatly enhanced BCC formation in parallel with the migration of tissue stem cells out of the bulge compartment. Finally, our group (4) assessed the cell of origin of tumors in the $\mathrm{Ptch}^{+/-}$mouse, which unperturbed forms minimal tumors but when stressed by ionizing or ultraviolet radiation forms microscopic and then visible BCCs.
Using K15-Cre and K14-Cre to conditionally remove specific DNA sequences from different cell populations, we found that BCCs in $P t c h 1^{+/-}$mice develop primarily from $\mathrm{K} 5^{+}$follicular stem cells as judged both directly, by fate mapping using an lslYFP allele that is expressed in cells in which Cre is activated and in their progeny, and indirectly, by stimulation of BCC carcinogenesis when p53 is deleted specifically from $\mathrm{K} 15^{+}$keratinocytes. In addition, we found that the interfollicular epidermis in our $\mathrm{Ptch}^{+/-}$mouse does not express immunohistochemically detectable Smo protein, thus providing an explanation as to why the presumed radiation-induced loss of the wild-type Ptch1 allele failed to induce tumors at this site. By contrast, when p53 was deleted from the interfollicular epidermis, Smo was expressed, and some BCCs developed at this site.

\section{A consensus view?}

First, for sure BCCs, at least in mice, arise from keratinocytes of the skin. Second, high-level Hh signaling without any additional imposed mutations can produce murine tumors, of at least microscopic size, that resemble human BCCs. Third, the details of the site of the cell of origin of murine BCCs can differ depending on the model used, and there is even some hint that different histologic patterns of BCCs might arise from different cells of origin (3). The models used all are artificial - for example, although ionizing radiation can predispose to human BCC carcinogenesis, epidemiology and the presence of signature mutations suggest that ultraviolet radiation plays a bigger role than does ionizing radiation in human BCCs, but it is uncertain whether ultraviolet radiation penetrates human skin as deeply as the site of the follicular bulge. The situation is further complicated by the movement of these cell populations - how best to define the cell of origin when tumors may not appear until the mutant cells have taken up residence in a site well removed from that in which it sustained its oncogenic mutation and perhaps had a different phenotype. Analogously, multipotent cerebellar stem cells that sustain an Hh activating mutation appear quite normal until they commit to a more limited state of differentiation $(11,12)$, and the cited work suggests that the bulge stem cell might have to "commit" to a more limited differentiation potential accompanying its migration to the secondary hair germ before it can develop into a BCC. And why do the keratinocytes expressing high-level Hh signaling become BCCs, whereas it appears that it is the cells expressing low levels of aberrant signaling that escape intrinsic tumor suppressor mechanisms and develop into cancers of the lung and breast (13-15)?

The true value of these five papers is evident in the fact that they raise more questions than they answer ... or, at least they suggest more avenues for further investigation.

\section{Acknowledgments}

This work was supported in part by CA115992.

Address correspondence to: Ervin Epstein, Children's Hospital of Oakland Research Institute, 5700 MLK Jr Way, Oakland, California 94609, USA. Phone: 510.502.6144; Fax: 510.597.7096; E-mail: eepstein@ chori.org.

1. Epstein EH. Basal cell carcinomas: attack of the hedgehog. Nat Rev Cancer. 2008;8(10):743-754.

2. Grachtchouk $M$, et al. Basal cell carcinomas in mice arise from hair follicle stem cells and multiple epithelial progenitor populations. J Clin Invest. 2011;121(5):1768-1781

3. Grachtchouk $M$, et al. Basal cell carcinomas in mice overexpressing Gli2 in skin. Nat Genet. 2000; 24(3):216-217.

4. Wang GY, WangJ, Mancianti ML, Epstein EHJr. Basal cell carcinomas arise from hair follicle stem cells in ptch1(+/-) mice. Cancer Cell. 2011;19(1):114-124.

5. Visvader JE. Cells of origin in cancer. Nature. 2011; 469(7330):314-322

6. Grachtchouk V, et al. The magnitude of hedgehog signaling activity defines skin tumor phenotype. EMBO J. 2003;22(11):2741-2751.

7. Youssef KK, et al. Identification of the cell lineage at the origin of basal cell carcinoma. Nat Cell Biol. 2010;12(3):299-305. 
8. Wong SY, Reiter JF. Wounding mobilizes hair follicle stem cells to form tumors. Proc Natl Acad Sci U S A 2011;108(10):4093-4098.

9. Kasper M, et al. Wounding enhances epidermal tumorigenesis by recruiting hair follicle keratinocytes. Proc Natl Acad Sci U S A. 2011;108(10):4099-4104.

10. Dolberg DS, Hollingsworth R, Hertle M, Bissell MJ Wounding and its role in RSV-mediated tumor formation. Science. 1985;230(4726):676-678.
11. Schuller $U$, et al. Acquisition of granule neuron precursor identity is a critical determinant of progenitor cell competence to form Shh-induced medulloblastoma. Cancer Cell. 2008;14(2):123-134.

12. Yang ZJ, et al. Medulloblastoma can be initiated by deletion of Patched in lineage-restricted progenitors or stem cells. Cancer Cell. 2008;14(2):135-145.

13. Sarkisian CJ, Keister BA, Stairs DB, Boxer RB, Moody SE, Chodosh LA. Dose-dependent onco- gene-induced senescence in vivo and its evasion during mammary tumorigenesis. Nat Cell Biol. 2007;9(5):493-505

14. Feldser DM, et al. Stage-specific sensitivity to p53 restoration during lung cancer progression. Nature. 2010;468(7323):572-575.

15. Junttila MR, et al. Selective activation of p53-mediated tumour suppression in high-grade tumours. Nature. 2010;468(7323):567-571.

\title{
Mitochondrial $\mathrm{Ca}^{2+}$ and ROS take center stage to orchestrate TNF- $\alpha$-mediated inflammatory responses
}

\author{
Laura A. Dada and Jacob I. Sznajder \\ Division of Pulmonary and Critical Care Medicine, Feinberg School of Medicine, Northwestern University, Chicago, Illinois, USA.
}

\begin{abstract}
Proinflammatory stimuli induce inflammation that may progress to sepsis or chronic inflammatory disease. The cytokine TNF- $\alpha$ is an important endotoxin-induced inflammatory glycoprotein produced predominantly by macrophages and lymphocytes. TNF- $\alpha$ plays a major role in initiating signaling pathways and pathophysiological responses after engaging TNF receptors. In this issue of $J C I$, Rowlands et al. demonstrate that in lung microvessels, soluble TNF- $\alpha$ (sTNF- $\alpha$ ) promotes the shedding of the TNF- $\alpha$ receptor 1 ectodomain via increased mitochondrial $\mathrm{Ca}^{2+}$ that leads to release of mitochondrial ROS. Shedding mediated by TNF- $\alpha$-converting enzyme (TACE) results in an unattached TNF receptor, which participates in the scavenging of sTNF- $\alpha$, thus limiting the propagation of the inflammatory response. These findings suggest that mitochondrial $\mathrm{Ca}^{2+}$, ROS, and TACE might be therapeutically targeted for treating pulmonary endothelial inflammation.
\end{abstract}

\section{Role of TNF- $\alpha$ in sepsis}

During sepsis, activation of proinflammatory pathways leads to dysfunction of mitochondria and cells, which contributes to multiorgan failure and poor outcomes. TNF- $\alpha$ simultaneously activates the JNK and NF-KB pathways and increases expression of the mitochondrial antioxidant protein SOD2, leading to an increase in $\mathrm{H}_{2} \mathrm{O}_{2}$ and the inactivation of JNK phosphatases. TNF receptors are differentially expressed in various cells and tissues and play a key role in modulating the cellular inflammatory responses (1). The severity of injury to the pulmonary endothelium during sepsis is determined by a complex interplay among proinflammatory cytokines

Conflict of interest: J.I. Sznajder is the editor of the American Journal of Respiratory and Critical Care Medicine and receives a stipend from the American Thoracic Society for this function.

Citation for this article: J Clin Invest. doi:10.1172/ JCI57748. (including TNF- $\alpha$ ), adhesion molecules expressed on the endothelial cells, and leukocytes recruited to the site of injury. Overwhelming sepsis or perturbation of the adaptive responses may lead to exacerbation of inflammation (2). An important mechanism to limit the TNF-mediated proinflammatory response involves shedding of the TNF- $\alpha$ receptor 1 (TNFR1) ectodomain, which is controlled by TNF- $\alpha-$ converting enzyme (TACE; also known as ADAM17), a disintegrin metalloproteinase $(3,4)$. Here, Rowlands et al. provide evidence that in the lung microvascular endothelium, administration of soluble TNF- $\alpha$ (sTNF- $\alpha$ ) increased cytosolic levels of $\mathrm{Ca}^{2+}$ via inositol1,4,5-triphosphate-mediated ( $\mathrm{IP}_{3}$-mediated) release from the ER (5). The increase in cytosolic $\mathrm{Ca}^{2+}$ was followed by a rapid increase in mitochondrial $\mathrm{Ca}^{2+}$, leading to a rise in mitochondrial ROS generation from complex III of the electron transport chain. These ROS were required for
TACE-mediated shedding of the TNFR1 ectodomain, which in turn modulated the endothelial inflammatory response.

\section{Mitochondrial function and $\mathrm{Ca}^{2+}$ signaling}

$\mathrm{Ca}^{2+}$ uptake by mitochondria is highly regulated, and the levels of mitochondrial $\mathrm{Ca}^{2+}$ play a central role in the metabolic (ATP production) and signaling (ROS production, release of proapoptotic molecules) functions of the organelle (Figure 1A). In patients with acute sepsis, mitochondrial function is impaired (Figure $1 B)$, and in patients with septic shock, the severity of impairment in mitochondrial function is associated with adverse clinical outcomes $(6,7)$. Normalization of mitochondrial biogenesis is necessary to restore oxidative metabolism during sepsis (Figure 1C) so that adequate $\mathrm{O}_{2}$ delivery and $\mathrm{O}_{2}$ tissue consumption can resume, allowing cellular metabolic needs to be met $(8,9)$.

Rowlands et al. suggest that the TNFmediated increase of mitochondrial $\mathrm{Ca}^{2+}$ is dependent on $\mathrm{ER} \mathrm{Ca}^{2+}$ release leading to $\mathrm{IP}_{3}$-mediated depletion of intracellular stores (5). It is known that store depletion triggers the activation of an inward rectifying $\mathrm{Ca}^{2+}$ current mediated either by the action of specialized $\mathrm{Ca}^{2+}$ releaseactivated (CRAC) channels or by the opening of voltage, receptor, or second messenger operated channels. TNF- $\alpha-$ induced increases in cytosolic $\mathrm{Ca}^{2+}$ lead to increased mitochondrial $\mathrm{Ca}^{2+}$ uptake through mitochondrial voltage-dependent anion channels, the mitochondrial 